\title{
Synthetic procedures
}

4-(Isopropyl-methyl-amino)benzonitrile (IMABN, $\mathrm{mp} 45.8-46.1{ }^{\circ} \mathrm{C}$ ) was obtained by reacting 4(isopropylamino)benzonitrile (IABN) with methyl iodide in methanol. IABN was made by refluxing a mixture of 4-aminobenzonitrile and isopropyl iodide (iPI) in methanol with $\mathrm{Na}_{2} \mathrm{CO}_{3} .{ }^{1} \mathrm{H}$ NMR for IMABN $\left(300 \mathrm{MHz}, \mathrm{CDCl}_{3}\right): 1.22\left(\mathrm{~d}, \mathrm{~N}-\mathrm{PPr}\left(\mathrm{CH}_{3}\right), \mathrm{J}=6.5 \mathrm{~Hz}\right), 2.82(\mathrm{~s}, \mathrm{~N}-\mathrm{Me}), 4.12(\mathrm{hpt}, \mathrm{N}-\mathrm{P} \operatorname{Pr}(\mathrm{CH}), \mathrm{J}=$ $6.5 \mathrm{~Hz}), 6.79(\mathrm{~d}, \mathrm{H} 3, \mathrm{~J}=8.8 \mathrm{~Hz}), 7.49(\mathrm{~d}, \mathrm{H} 2, \mathrm{~J}=8.8 \mathrm{~Hz})$.

4-(T-butyl-methyl-amino)benzonitrile (tBMABN, mp 73.0-73.4 ${ }^{\circ} \mathrm{C}$ ) was synthesized from 4-(tbutylamino)benzonitrile (tBABN) and methyl iodide in methanol. tBABN was made by refluxing a mixture of 4-aminobenzonitrile and t-butyl iodide (tBuI) in methanol with $\mathrm{Na}_{2} \mathrm{CO}_{3} .{ }^{1} \mathrm{H} \mathrm{NMR}$ of tBMABN (300 MHz, $\mathrm{CDCl}_{3}$ ): 1.26 (s, N-tBu), 2.88 (s, N-Me), 7.09 (d, H3, J = 8.4 Hz), 7.49 (d, H2, J = 8.4 Hz). NIC6 was made in four steps. ${ }^{1}$ 1,2,3,4-tetrahydroquinoline was reacted with isopropyl iodide, giving 1-isopropyl-1,2,3,4-tetrahydroquinoline. This compound was converted to 1-isopropyl-1,2,3,4tetrahydroquinoline-6-carbaldehyde by insertion into a mixture of dimethylformamide and $\mathrm{POCl}_{3}$. $\mathrm{From}$ the carbaldehyde, the corresponding oxime was made with hydroxylamino hydrochloride. Finally, NIC6 (mp 81.5-82.0 ${ }^{\circ} \mathrm{C}$ ) was obtained by refluxing the oxime in acetic anhydride. ${ }^{1} \mathrm{H}$ NMR for NIC6 (300 $\left.\mathrm{MHz}, \mathrm{CDCl}_{3}\right): 1.19\left(\mathrm{~d}, \mathrm{~N}-\mathrm{iPr}\left(\mathrm{CH}_{3}\right), \mathrm{J}=6.5 \mathrm{~Hz}\right), 1.86(\mathrm{~m}, \mathrm{H} 3), 2.68(\mathrm{t}, \mathrm{H} 4, \mathrm{~J}=6.2 \mathrm{~Hz}), 3.21(\mathrm{t}, \mathrm{H} 2, \mathrm{~J}=$ $5.8 \mathrm{~Hz}), 4.09$ (hpt, N-iPr(CH), J = 6.5 Hz), 6.59 (d, H8, J = 9.1 Hz), 7.13 (qt, H5), 7.27 (dd, H7, J = 9.1/2.3 Hz).

NMC5 (mp 38.8-39.7 ${ }^{\circ} \mathrm{C}$; litt.: $\left.{ }^{2} 34.0-35.0^{\circ} \mathrm{C}\right)$, 1-ethyl-5-cyano-indoline (NEC5, mp 71.1-71.7 ${ }^{\circ} \mathrm{C}$; litt.: ${ }^{3}$ $\left.67.5{ }^{\circ} \mathrm{C}\right), \quad$ NIC5 (mp 75.4-75.7 $\left.{ }^{\circ} \mathrm{C}\right)$, NMC6 (mp 53.9-54.1 $\left.{ }^{\circ} \mathrm{C}\right)$ and 1-ethyl-6-cyano-1,2,3,4tetrahydroquinoline (NEC6, mp 73.9-74.2 ${ }^{\circ} \mathrm{C}$ ) were synthesized in a comparable manner, starting from indoline (NXC5) and 1,2,3,4-tetrahydroquinoline (NXC6), respectively. ${ }^{1} \mathrm{H}$ NMR for NMC5 (300 MHz, $\left.\mathrm{CDCl}_{3}\right): 2.83(\mathrm{~s}, \mathrm{Me}), 2.99(\mathrm{t}, \mathrm{H} 3, \mathrm{~J}=8.55 \mathrm{~Hz}), 3.49(\mathrm{t}, \mathrm{H} 2, \mathrm{~J}=8.55 \mathrm{~Hz}), 6.34(\mathrm{~d}, \mathrm{H} 6, \mathrm{~J}=8.23 \mathrm{~Hz})$, 7.22 (q, H4), 7.35 (ddt, 7H, J = 8.2/1.7/0.65 Hz). ${ }^{1} \mathrm{H}$ NMR for NEC5 (300 MHz, $\left.\mathrm{CDCl}_{3}\right): 1.17(\mathrm{t}$, ethyl $\left.\left(\mathrm{CH}_{3}\right), \mathrm{J}=7.9 \mathrm{~Hz}\right), 3.00(\mathrm{t}, \mathrm{H} 3, \mathrm{~J}=8.65 \mathrm{~Hz}), 3.23\left(\mathrm{q}\right.$, ethyl $\left.\left(\mathrm{CH}_{2}\right), \mathrm{J}=7.9 \mathrm{~Hz}\right), 3.52(\mathrm{t}, \mathrm{H} 2, \mathrm{~J}=8.65$ 
Hz), 6.33 (d, H6, J = 8.2 Hz), 7.21 (q, H4, J = 1.8 Hz), 7.33 (d, H7, J = 8.2 Hz). ${ }^{1} \mathrm{H}$ NMR (300 MHz,

$\mathrm{CDCl}_{3}$ ) for NIC5: $1.16\left(\mathrm{~d}, \mathrm{~N}-\mathrm{iPr}\left(\mathrm{CH}_{3}\right), \mathrm{J}=6.7 \mathrm{~Hz}\right), 2.95(\mathrm{t}, \mathrm{H} 3, \mathrm{~J}=8.65 \mathrm{~Hz}), 3.49(\mathrm{t}, \mathrm{H} 2 \mathrm{~J}=8.65 \mathrm{~Hz})$, 3.82 (hpt, N-iPr(CH), J = 6.7 Hz), 6.29 (d, H7, J = 8.3 Hz), 7.17 (qt, H4), 7.30 (dd, H7, J = 8.4/1.75

Hz). ${ }^{1} \mathrm{H}$ NMR for NMC6 (300 MHz, $\left.\mathrm{CDCl}_{3}\right): 1.95$ (m, H3), 2.73 (t, H4, J = 6.2 Hz), 2.95 s, N-CH $)_{3}$, $3.35(\mathrm{t}, \mathrm{H} 2, \mathrm{~J}=5.6 \mathrm{~Hz}), 6.48(\mathrm{~d}, \mathrm{H} 8, \mathrm{~J}=8.5 \mathrm{~Hz}), 7.15(\mathrm{q}, \mathrm{H} 5,2.0 / 1.0 \mathrm{~Hz}), 7.32(\mathrm{dd}, \mathrm{H} 7, \mathrm{~J}=8.5 / 2.1$ Hz). ${ }^{1} \mathrm{H}$ NMR for NEC6 (300 MHz, $\left.\mathrm{CDCl}_{3}\right): 1.16\left(\mathrm{t}\right.$, ethyl $\left.\left(\mathrm{CH}_{3}\right), \mathrm{J}=7.15 \mathrm{~Hz}\right), 1.94(\mathrm{~m}, \mathrm{H} 3), 2.72(\mathrm{t}, \mathrm{H} 4$, $\mathrm{J}=6.3 \mathrm{~Hz}), 3.34(\mathrm{~m}, \mathrm{H} 2), 3.37$ (q, ethyl $\left.\left(\mathrm{CH}_{2}\right), \mathrm{J}=7.15 \mathrm{~Hz}\right), 6.52$ (d, H7, J = 8.6 Hz), 7.16 (qt, H5), $7.29(\mathrm{dd}, \mathrm{H} 8, \mathrm{~J}=8.6 / 2.1 \mathrm{~Hz})$. The ${ }^{1} \mathrm{H}$ and ${ }^{13} \mathrm{C}$ NMR spectra were measured with Varian Unity-300, Varian Inova 500, Varian Inova 600, Bruker AMX 300 and Varian Mercury spectrometers.

The procedure employed here to make the NXC5 and NXC6 with methyl (M), ethyl (E) and isopropyl (I) substituents did not lead to success in the case of the t-butyl derivative NTC6, however. Therefore, a number of different procedures were followed on the way to the synthesis of this compound, as described in the following. 1,2,3,4-Tetrahydroquinoline was reacted with t-butyl iodide (tBuI) in water with $\mathrm{K}_{2} \mathrm{CO}_{3}$ for 18 hours at $60{ }^{\circ} \mathrm{C}$. As a result of this reaction, instead of NTC6 a small amount (a yield of around 3 percent) of 8-t-butyl-1,2,3,4-tetrahydroquinoline (8tBTHQ) was obtained. The unexpected position of the t-butyl group in the position 8 of the aromatic ring was established by NOE and HMBC experiments. ${ }^{1} \mathrm{H}$ NMR for 8tBTHQ (300 MHz, $\left.\mathrm{CDCl}_{3}\right): 1.40(\mathrm{~s}, 8-\mathrm{tBu}), 1.93(\mathrm{~m}, \mathrm{H} 3, \mathrm{~J}=6.6 \mathrm{~Hz}), 2.82$ (t, H4, J = 6.52 Hz), $3.38(\mathrm{t}, \mathrm{H} 2, \mathrm{~J}=5.69 \mathrm{~Hz}), 4.05(\mathrm{HN}), 6.58(\mathrm{t}, \mathrm{H} 6, \mathrm{~J}=7.67 \mathrm{~Hz}), 6.86(\mathrm{ddt}, \mathrm{H} 5, \mathrm{~J}=$ 7.4/1.54/0.8 Hz), $7.07(\mathrm{dd}, \mathrm{H} 7, \mathrm{~J}=7.89 / 1.54 \mathrm{~Hz}) .{ }^{13} \mathrm{C} \mathrm{NMR}\left(50.3 \mathrm{MHz}, \mathrm{CDCl}_{3}\right): 21.93(\mathrm{t}, \mathrm{C} 3), 27.99(\mathrm{t}$, C4), $29.86\left(\mathrm{q}, \mathrm{tBu}\left(\mathrm{CH}_{3}\right)\right), 34.0(\mathrm{~s}, \mathrm{tBu}(\mathrm{C})), 42.15$ (t, C2), 116.12 (d, C6), 122.07 (s, C10), 124.06 (d, C7), 127.87 (d, C5), 132.37 (s, C8), 143.02 (s, C9).

It was then attempted to synthesize NTC6 starting from quinoline and tBuBr, via 1-t-butyl-quinolium as the intended intermediate, which was then to be converted to NTC6 by a reaction with formic acid $(\mathrm{HCOOH})$ and triethylamine. ${ }^{4}$ Although a reaction between quinoline and $\mathrm{tBuBr}$ was seen to take place, 
the quinolinium cation with a $\mathrm{H}$ atom instead of a t-butyl group attached to the nitrogen is produced, as established by NMR and elemental analysis. Possibly, the initially present t-butyl group was transformed into isobutene. A reaction between quinoline and $\mathrm{tBuI}\left(40^{\circ} \mathrm{C}, 16\right.$ hours $)$ led to the same result.

\section{Scheme 1. Synthesis of NTC6.}
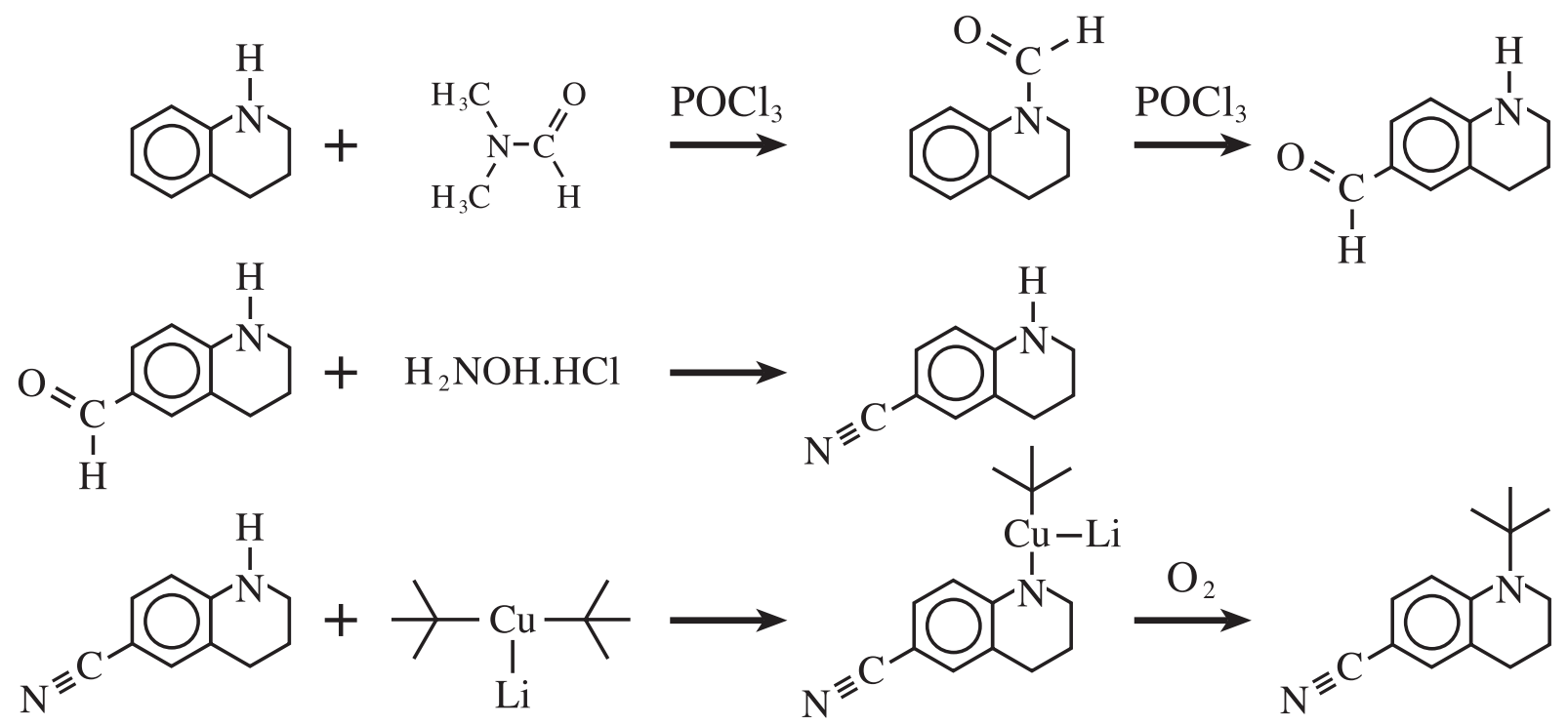

The final and successful procedure to obtain NTC6 (Scheme 1) is described in the main text.

${ }^{1} \mathrm{H}$ NMR of NTC6 (300 MHz, CDCl $): 1.45(\mathrm{~s}, \mathrm{~N}-\mathrm{tBu}), 1.82(\mathrm{~m}, \mathrm{H} 3), 2.56(\mathrm{t}, \mathrm{H} 4, \mathrm{~J}=6.1 \mathrm{~Hz}), 3.31(\mathrm{t}$, $\mathrm{H} 2, \mathrm{~J}=6.3 \mathrm{~Hz}), 6.93(\mathrm{~d}, \mathrm{H} 8, \mathrm{~J}=8.8 \mathrm{~Hz}), 7.15(\mathrm{~m}, \mathrm{H} 5), 7.25(\mathrm{dd}, \mathrm{H} 7, \mathrm{~J}=8.8 / 2.1 \mathrm{~Hz}) .{ }^{13} \mathrm{C} \mathrm{NMR}: 24.19$ (t, C3), 28.63 (t, C4), 28.69 (q, N-tBu(CH$)$ ), 44.74 (t, C2), 56.00 (s, N-tBu(C)), 97.20 (s, C6), 116.34 (d, C8), 120.90 (s, CN), 128.70 (s, C10), 130.18 (d, C7), 131.16 (d, C5), 148.97 (s, C9).

NTC5 was synthesized in a similar manner: 2,3-dihydro-1H-indole-5-carbonitrile (NHC5) was made from 5-cyanoindole in a reaction with bis(trifluoroacetoxy)borane-THF, BH3.THF and CF3COOH. ${ }^{5}$ NHC5 in THF was added slowly to a suspension consisting of $\mathrm{CuI}$ and t-butyllithium in THF at $-60{ }^{\circ} \mathrm{C}$. After stirring for 2 hours, excess oxygen was inserted into the solution for 10 minutes, during which time the temperature of the cooled solution rises to $-54{ }^{\circ} \mathrm{C}$. The solution obtained by this oxidative coupling of lithium alkylcopper amide was poured into a $25 \%$ ammonia solution and extracted with 
ethyl acetate. ${ }^{6}$ The product was purified by flash chromatography and HPLC, giving NTC5. ${ }^{1} \mathrm{H}$ NMR $\left(300 \mathrm{MHz}, \mathrm{CDCl}_{3}\right)$ of NTC5: $1.36(\mathrm{~s}, \mathrm{~N}-\mathrm{tBu}), 2.88(\mathrm{t}, \mathrm{H} 3, \mathrm{~J}=8.7 \mathrm{~Hz}), 3.55(\mathrm{t}, \mathrm{H} 2, \mathrm{~J}=8.7 \mathrm{~Hz}), 6.65(\mathrm{~d}$, $\mathrm{H} 7, \mathrm{~J}=8.4 \mathrm{~Hz}), 7.17(\mathrm{q}, \mathrm{H} 4), 7.26(\mathrm{dd}, \mathrm{H} 6, \mathrm{~J}=8.4 / 1.8 \mathrm{~Hz})$.

\section{References}

(1) Visser, R. J.; Varma, C. A. G. O. J. Chem. Soc., Faraday Trans. 2 1980, 76, 453.

(2) Rotkiewicz, K.; Grabowski, Z. R.; Krówczyński, A.; Kühnle, W. J. Lumin. 1976, 12/13, 877.

(3) Rettig, W.; Rotkiewicz, K.; Rubaszewska, W. Spectrochim. Acta 1984, 40A, 241.

(4) Kost, A. N.; Yudin, L. G. J. Gen. Chem. USSR 1956, 26, 1929.

(5) Maryanoff, B. E.; McComsey, D. F.; Nortey, S. O. J. Org. Chem. 1981, 46, 355.

(6) Yamamoto, H.; Maruoka, K. J. Org. Chem. 1980, 45, 2739.

\section{Table of solvent properties}

Table. Solvent Polarity Parameters

\begin{tabular}{lllllll}
\hline Solvent & $\varepsilon$ & $n$ & $f(\varepsilon)^{\mathrm{a}}$ & $f\left(n^{2}\right)^{\mathrm{b}}$ & $f(\varepsilon)-f\left(n^{2}\right)$ & $f(\varepsilon)-1 / 2 f\left(n^{2}\right)$ \\
\hline$n$-hexane (1) & 1.88 & 1.372 & 0.185 & 0.185 & 0 & 0.092 \\
cyclopentane (2) & 1.96 & 1.404 & 0.195 & 0.197 & -0.001 & 0.097 \\
$n$-hexadecane (3) & 2.05 & 1.435 & 0.205 & 0.207 & -0.001 & 0.102 \\
cis-decaline (4) & 2.21 & 1.479 & 0.223 & 0.221 & 0.002 & 0.113 \\
di(n-hexyl) ether (5) & 2.75 & 1.420 & 0.269 & 0.202 & 0.067 & 0.168 \\
di(n-pentyl) ether (6) & 2.86 & 1.412 & 0.277 & 0.199 & 0.077 & 0.177 \\
di(n-butyl) ether (7) & 3.05 & 1.398 & 0.289 & 0.194 & 0.095 & 0.192
\end{tabular}




$\begin{array}{lllllll}\text { di(n-propyl) ether (8) } & 3.26 & 1.379 & 0.301 & 0.188 & 0.113 & 0.207 \\ \text { diethyl ether (9) } & 4.24 & 1.350 & 0.342 & 0.177 & 0.165 & 0.253 \\ n \text {-butyl acetate (10) } & 4.95 & 1.392 & 0.362 & 0.192 & 0.170 & 0.266 \\ n \text {-propyl acetate (11) } & 5.52 & 1.382 & 0.375 & 0.189 & 0.187 & 0.281 \\ \text { ethyl acetate (12) } & 5.99 & 1.370 & 0.384 & 0.184 & 0.200 & 0.292 \\ \text { methyl acetate (13) } & 6.88 & 1.358 & 0.398 & 0.180 & 0.218 & 0.308 \\ \text { tetrahydrofuran (14) } & 7.39 & 1.405 & 0.405 & 0.197 & 0.208 & 0.307 \\ \text { dichloromethane (15) } & 8.87 & 1.421 & 0.420 & 0.202 & 0.218 & 0.319 \\ \text { 1,2-dichloroethane (16) } & 10.4 & 1.443 & 0.431 & 0.210 & 0.221 & 0.326 \\ n \text {-butyl cyanide (17)) } & 19.7 & 1.395 & 0.463 & 0.193 & 0.269 & 0.366 \\ n \text {-propyl cyanide (18) } & 24.2 & 1.382 & 0.470 & 0.189 & 0.281 & 0.375 \\ \text { ethyl cyanide (19) } & 29.2 & 1.364 & 0.475 & 0.182 & 0.293 & 0.383 \\ \text { methyl cyanide (20) } & 36.7 & 1.342 & 0.480 & 0.174 & 0.306 & 0.393 \\ \text { methanol (21) } & 32.7 & 1.326 & 0.477 & 0.168 & 0.309 & 0.393\end{array}$

$$
{ }^{\mathrm{a}} f(\varepsilon)=(\varepsilon-1) /\left(2 \varepsilon^{+}+1\right) .{ }^{\mathrm{b}} f\left(n^{2}\right)=\left(n^{2}-1\right) /\left(2 n^{2}+1\right) .
$$

